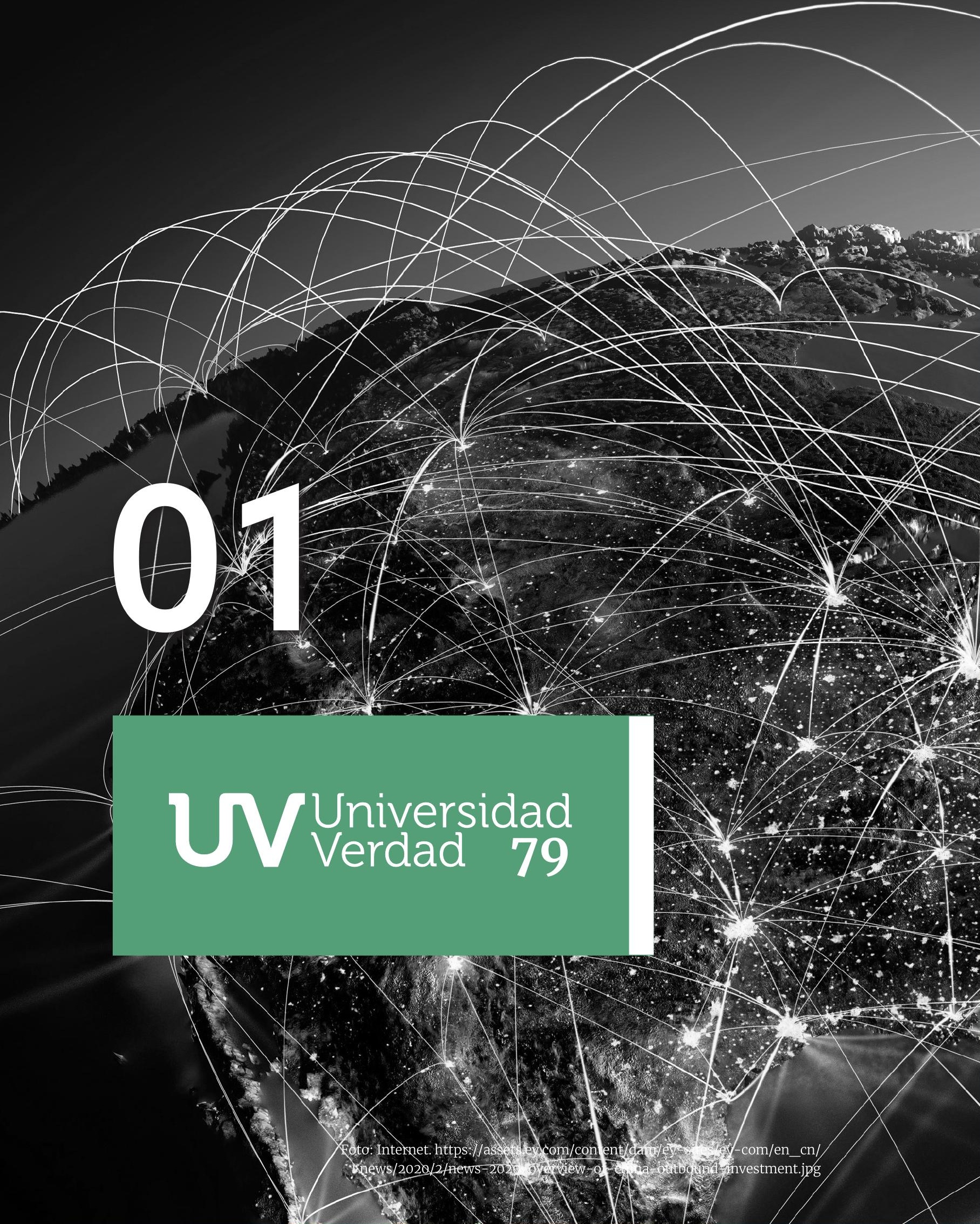




\title{
TRANSPORTE, MOVILIDAD Y ACCESIBILIDAD: CAMPOS Y MÉTODOS EMERGENTES PARA EL ANÁLISIS GEOGRÁFICO CONTEMPORÁNEO
}

\section{Transportation, mobility and accessibility: Emerging fields and methods for contemporary geographic analyses}

\author{
iD Alejandro Cortés Salinas, Universidad Alberto Hurtado (Chile) \\ (alcortes@uahurtado.cl) (https://orcid.org/0000-0002-2680-2621) \\ (iD Loreto Rojas-Symmes, Universidad Alberto Hurtado (Chile) \\ (lorojas@uahurtado.cl) (https://orcid.org/0000-0001-5022-481X)
}

\section{Resumen}

El transporte, la movilidad y la accesibilidad son hoy campos relevantes para comprender la relación sinérgica que existe entre los sistemas de desplazamiento, la distribución de la oferta de bienes y servicios y los comportamientos que los ciudadanos realizan (o no) en sus territorios.

El presente trabajo, a partir de una revisión bibliográfica, da cuenta de la relevancia de estos temas para disciplinas como la geografía, así como la pertinencia de los métodos para el abordaje de desafíos en el ámbito de la investigación académica, como del diseño y operación de sistemas de transporte, planes de infraestructura o instrumentos de ordenamiento territorial.

De forma complementaria, el trabajo releva la nueva oportunidad de reflexionar respecto de estos campos temáticos, destacando sus tensiones, restricciones y mutaciones, a la luz de ciudades que crecen y se complejizan cada vez más rápidamente, instalando nuevos desafíos en la concreción de desarrollos urbanos más accesibles y sostenibles.

\section{Palabras clave}

Transporte, movilidad, accesibilidad, análisis espacial.

\section{Keywords}

Transportation, Mobility, Accessibility, Spatial Analysis.

\begin{abstract}
Transportation, mobility and accessibility are relevant fields for understanding the synergetic between travel systems, the distribution of the supply of goods and services and the behavior of citizens in their territories. This work, based on a bibliographic review, shows the relevance of these issues for disciplines such as geography, as well as the relevance of the methods for addressing challenges in the field of academic research, such as design and operation of transportation systems, infrastructure plans or instruments of land use planning.

Additionally, this work proposes to reflect on these thematic fields, highlighting their tensions, restrictions and mutations, in view that cities grow and become increasingly complex, generating new challenges in the realization of more accessible and sustainable urban developments.
\end{abstract}


1.

\section{Introducción: la importancia de rele- var los estudios de transporte, movi- lidad y accesibilidad en el análisis de territorios contemporáneos}

Uno de los desafíos a los que se enfrentan las ciudades contemporáneas es el de la movilidad cotidiana (Hernández, 2012). En un contexto en el que para realizar varias actividades se debe recorrer importantes distancias y, por ende, utilizar medios motorizados, la discusión acerca del rol del transporte, la movilidad y la accesibilidad es algo ineludible.

En América Latina y El Caribe el transporte constituye uno de los elementos principales en la canasta de bienes y servicios que consumen los hogares (Gandelman, et al. 2019). El 44\% de la población en las ciudades más grandes de la región viaja 1,5 horas por día, con un costo económico equivalente a 10 semanas laborales/año/persona (BID, 2014).

Además, según el Banco Mundial (2019), la proporción del transporte en el consumo total es mucho mayor que en cualquier otra región en desarrollo en todo el mundo, así como la región en desarrollo con la mayor proporción del gasto familiar total dedicado al transporte $(17 \%$, en contraste con $9 \%$ en África Subsahariana, $11 \%$ en Europa oriental y Asia central o 5\% en Asia meridional), lo que compromete la capacidad de los hogares para acceder al transporte.
Si bien, la creciente multiplicación de los viajes sólo puede verse como un reflejo de la realización de oportunidades de conexión, el desafío actual debe estar en garantizar redes de conexión y transporte adecuadas a las formas de movilidad que requieren los diferentes grupos sociales (Herce, 2009). Esto, a pesar de que estamos ante un escenario donde las mayores inversiones en infraestructura de transporte son en carreteras (Banco Mundial, 2015) y el automóvil es valorado como el modo más eficiente para los desplazamientos urbanos motorizados (Cortés, et al. 2016).

De esta forma es posible constatar que las políticas públicas si bien han incorporado a la movilidad como un desafío en las agendas urbanas, las acciones propuestas continúan enfocándose en aumentar las velocidades de los desplazamientos y reducir la congestión, con expresiones como la priorización de proyectos de vías expresas o de alta capacidad (autopistas) por sobre el transporte no motorizado y público, tal como ya fue comentado anteriormente. Esto, en lugar de mejorar las condiciones de acceso a los bienes y servicios urbanos, es probable que exacerbe los altos niveles de segregación y la desigualdad social y espacial, dificultando la capacidad de poder cumplir con el Objetivo de Desarrollo Sostenible 11.2 de proporcionar "acceso a sistemas de transporte seguros, asequibles, accesibles y sostenibles para todos" (UN Habitat, 2016).

Disciplinalmente, desde la geografía el abordaje teórico y metodológico de estas temáticas ha concentrado tradicionalmente sus esfuerzos en comprender el desplazamiento de las personas, la carga, la información y su organización espacial, considerando los atributos y restricciones 
relacionados con el origen, destino, extensión, naturaleza y propósito de los movimientos (Rodrigue, 2017).

Sin embargo, desde la década de 2000 es posible constatar cómo desde la disciplina se pone en tensión la funcionalidad propia del quehacer científico del siglo XX con las nuevas propuestas de sostenibilidad, movilidad y accesibilidad. Tras una extensa revisión de la relación entre transporte y ciudad, Miralles (2002) sostiene que la geografía del transporte puede definirse como el estudio de los sistemas de transporte y sus impactos territoriales. El interés, por tanto, se encuentra en los medios que permiten el desplazamiento, así como también en las dinámicas que se establecen. Esto permite que actualmente, a partir de los múltiples métodos de análisis espacial desarrollados (tanto cualitativos, cuantitativos como de geotecnologías), la geografía del transporte sea un campo de crecientes requerimientos tanto para complementar estudios de transporte interdisciplinares, como también para aportar de forma autónoma a la planificación y operación de distintos sistemas de desplazamiento.

Complementariamente, desde la década de 1980 surge la preocupación por la vinculación del transporte con procesos sociales, lo que algunos autores definen como la dimensión "macro" de la movilidad (Widmer y Schneider, 2006), al vincular al transporte con los cambios socioespaciales de las sociedades. Estos estudios tendrán planteamientos complejos, superando el análisis locacional- causal, donde un elemento clave para entender la relación entre transporte y espacio es la escala, poniéndose especial énfasis en el problema estructural de las políticas de transporte. Miralles (2002), describe al arribo de los estudios de movilidad como "un nuevo paradigma" cuyo énfasis está en la comprensión de las movilidades diferenciales, definiendo al transporte como el soporte material de la movilidad, y dejando en evidencia la preocupación por la comprensión de los viajes y sus motivaciones, y entendiendo así a la movilidad como una relación social donde el centro (objeto de estudio) es el individuo.

De forma paralela, desde la década de 1960 la geografía se interesó por los estudios de accesibilidad, los cuales incorporan elementos relacionales, interesándose por la oferta de movilidad, asociándola con la limitaciones o potencialidades (en términos de condición necesaria), para el ejercicio de la movilidad, o bien, como la "dimensión espacial" de la movilidad, aspecto que se revisa con mayor detención en los siguientes apartados.

2.

\section{Del transporte a la movilidad: vínculo sinérgico clave para comprender las relaciones espaciales en la cotidiani- dad}

El concepto de movilidad está íntimamente vinculado con el de transporte. En las investigaciones sobre geografía del transporte, la movilidad generalmente es referida como una medida de comportamiento cuya comprensión se basa en el conjunto de desplazamientos que efectivamente realizan las personas en el espacio, cualquiera sea su duración, distancia del desplazamiento, medios usados, causas y/o consecuencias (Módenes, 2007).

Sin embargo, en la sociedad actual, la movilidad de las personas ha adquirido una importancia muy superior a la que tenía en períodos anteriores de la ciudad contemporánea (Herce, 2009). Hoy, las decisiones de localización se adoptan a menudo por el potencial de posibles relaciones que ofrece un territorio determinado. Pero, a diferencia de lo propuesto en los modelos locacionales de geografía del transporte de la segunda mitad del siglo XX, la movilidad exige superar el determinismo de los factores de localización de las actividades y comprender más bien desde criterios de oportunidad, oferta de externalidades, competencia entre espacios, especialización territorial, mutación constante de usos del territorio, etc.

De esta forma, los estudios de movilidad se relacionan con la forma en que las personas organizan sus desplazamientos sobre el espacio urbano. Analizar los efectos territoriales desde esta dimensión "implica centrar la atención en la 
satisfacción de las necesidades de conexión, que incluyen motivos muy variados y con alteración frecuente de su expresión espacial y temporal" (Herce, 2009, p. 20). En la movilidad descansa la realización de las oportunidades de desarrollo personal y social de la población- porque estamos en una sociedad de conexiones a muy distinto nivel-, por lo que pierden sentido las clasificaciones que suponen una categorización jerárquica de esos motivos.

Lo anterior no sólo remite a la conectividad, asociada a la materialidad de las redes; sino también a la sociabilidad, vinculada a la cohesión, la integración y la identidad. En este sentido, "la movilidad es resultante de la distribución territorial de infraestructuras y servicios de transporte, y también de aspectos que se enmarcan en lo familiar, vecinal, y social" (Gutiérrez, 2010).

De esta forma, los efectos urbanos relacionados con la movilidad se enfrentan a una polisemia que en parte responde a distintos ángulos o dimensiones de abordaje: la movilidad considerada como un valor, un derecho, una capacidad, un conjunto de competencias o un capital social, entre otras (Ascher, 2005; Kaufmann, 2008; Le Breton, 2005; Urry, 2002).

Un punto de partida común de todos estos abordajes supone que los individuos buscan tener autonomía y control de espacio-tiempos de su vida cotidiana, así como la posibilidad de ampliar la esfera de la intimidad o de sus interacciones sociales. De esta forma, los efectos del transporte en la organización de los desplazamientos condicionan la facilidad de acceso a la vivienda, al trabajo, la educación, cultura, salud, así como también al amor, la amistad o la política (Ascher, 2005).

Sin embargo, en la ciudad contemporánea esta movilidad "a medida" en la mayoría de los casos es convertida según Kaufmann (2002) en movimientos recursivos, no sólo por su reiteración sino porque la repetición es fuente de construcción de hábitos espaciales, de evidente constatación a partir de las estrategias de movilidad.
Por tanto, los efectos no sólo están referidos a la forma en que se realizan los desplazamientos, sino también a las razones o motivaciones que posibilitan o no la realización de un desplazamiento dado y el porqué de la forma en que se efectúa, lo cual da cuenta de una elección bajo un conjunto de opciones y de restricciones de posible ejecución. Esto es relevante porque implica múltiples opciones de ejecutar un desplazamiento, pero que, para un ciudadano, cuyos recursos son siempre limitados, sus opciones se restringen en muchos casos sólo a una, que es la que finalmente ejecuta, razón por la que la intervención de este espacio cotidiano puede resultar trascendentalmente sensible.

En la última década los sistemas de movilidad están siendo analizados desde nuevos enfoques, más allá de la tradicional concepción ingenieril de las infraestructuras y el transporte (López y Ravella, 2019). En este sentido, la geografía cobra especial relevancia al ser una disciplina que busca explicar las relaciones espaciales, siendo las redes de transporte uno de los principales soportes físicos de estas interacciones (Rodrigue, 2017), las que operan hoy en una amplia gama de escalas e, inclusive de formas superpuestas que van desde el individuo, el hogar, el barrio, la ciudad, la región, el estado y/o lo global (Curl y Davison, 2014).

\section{3.}

\section{De la movilidad a la accesibilidad: hacia la construcción de desarrollos urbanos más accesibles en la ciudad contemporánea}

Complementando la definición de Módenes (2007) sobre la movilidad como una medida de comportamiento, la accesibilidad es esencialmente una medida de potencial de desplazamiento para alcanzar las oportunidades espacialmente distribuidas de empleo, ocio, interacción social u otras (Hodge, 1997; Páez, et al. 2012). Es una medida cada vez más utilizada para medir la sostenibilidad del transporte al reconocer sus tres dimensiones: eficiencia, medio ambiente y equidad social (Chen y Akar, 2017). 
De hecho, es cada vez más común ver que en las agendas urbanas, tanto globales como nacionales o locales, se plantee que uno de los objetivos clave de la planificación del transporte urbano es proporcionar a las personas acceso a un mayor número de oportunidades para la interacción con otras personas y lugares, un objetivo que apoya la dinámica creativa, la habitabilidad y la productividad de las ciudades (Campbell, et al. 2019).

La accesibilidad puede ser entendida como el principal "producto" del sistema de transporte (Zondag, et al. 2015). Por ejemplo, ante la construcción de una nueva infraestructura de transporte, se producen cambios inmediatos en la accesibilidad del territorio, los cuales trascienden a la reducción de los tiempos de viajes o la creación de ventajas competitivas para un lugar determinado (CondeçoMelhorado, et al. 2011; Calvo-Poyo, et al. 2019).

Medir la accesibilidad supone poner atención en un enfoque de oferta de espacios e infraestructuras adaptadas a cada forma de desplazamiento en el comportamiento espacial de la movilidad (Herce, 2009). Esto, porque el espacio urbano es un espacio limitado, con múltiples demandas, y serán políticas de decisión de la oferta disponible de los diferentes espacios adaptados a los distintos tipos de movilidad los que marquen el modo de organización de la ciudad. Simular cambios en la accesibilidad permite determinar qué operaciones previstas (de futuras obras) tendrían mayor utilidad en la consecución de objetivos de ordenamiento territorial.

El análisis riguroso de accesibilidad tiene un potencial poco desarrollado como instrumento de apoyo a la toma de decisiones tanto en el proceso de planificación de infraestructuras de transporte como su evaluación ex post (López, et al. 2009; Monzón, et al. 2010; Silva, et al. 2017).

De hecho, en Montreal (Canadá), la elección de desarrollar infraestructuras de transporte público ha estado basada en la evaluación de la accesibilidad ofrecida por el sistema de transporte público en comparación con la ofrecida por el automóvil privado (El-Geneidi, et al. 2011). Sin embargo, son aún pocos los estudios que miden la accesibilidad en ciudades en países de ingresos bajos (Campbell, et al. 2019).
En términos procesuales, la accesibilidad es un constructo de tres componentes: transporte, uso de suelo y de individuos (Niedzielski y Boschmann, 2014), los cuales se desarrollan en dos dimensiones: en el tiempo y en el espacio (Geurs y Van Wee, 2004). En función de los objetivos del análisis cada componente tendrá más peso sobre otro $\mathrm{y}$, del mismo modo, las variables, indicadores, formulaciones y fuentes necesarias dependerán asimismo de estos componentes (Martínez, 2012). Algunas de estas interacciones se presentan en la Figura 1. 


\section{Figura 1}

Componentes de la accesibilidad e interacciones

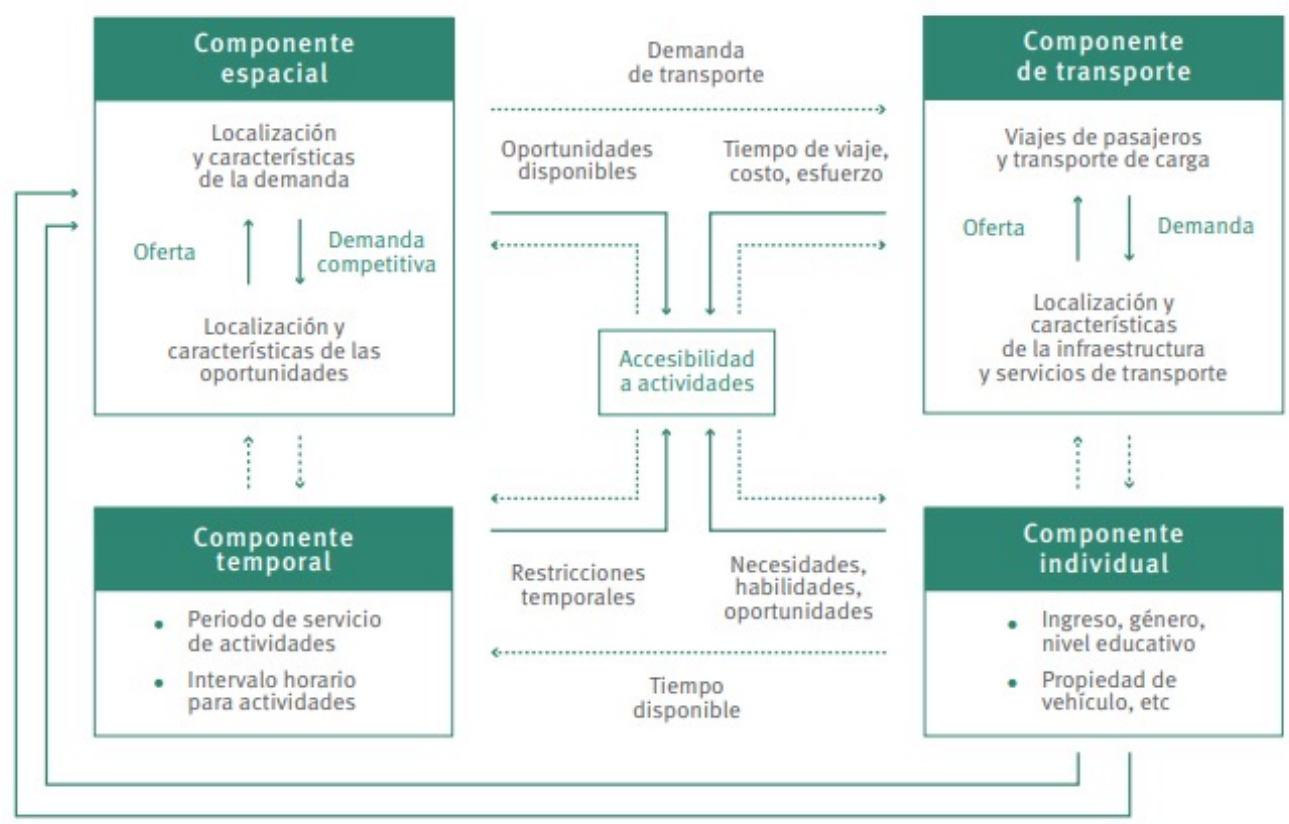

Fuente: BID (2020) adaptado de Geurs y Van Wee (2004)

De esta forma, la accesibilidad resume el efecto que la provisión y/o gestión de las infraestructuras de transporte generan sobre la productividad y el bienestar de las interacciones económicas y sociales que ocurren en el espacio urbano (Calvo-Poyo, et al. 2019; Graham, 2014; López, et al. 2009). Una mejora en la accesibilidad implica una reducción en las distancias reales entre las personas y las actividades, lo que potencia los beneficios de la aglomeración (CAF, 2017). Genera un impacto directo sobre la cohesión territorial, al aumentar el bienestar de las familias que se benefician de un mejor acceso al empleo, la salud, educación y/o servicios en general, así como las empresas al reducir los costos de transporte en que incurren sus procesos productivos (Bertolini, et al. 2005)

Esto, independiente de los niveles de densidad habitacional o residencial, ya que la accesibilidad podría verse disminuida en ciudades con alta densidad, pero con altos costos de congestión. También impactará sobre las pautas del desarrollo urbano, en la medida en que la distribución de las mejoras de accesibilidad sea desigual y cambiante sobre el espacio urbano (Gutiérrez, 2001). Por ello, el debate de las políticas públicas no debería estar centrado en si las ciudades deben promover modelos de desarrollo más o menos compactos, sino en cómo generar modelos de desarrollo urbano más accesibles (CAF, 2017, p. 58).

Puede ser vista como un proxy para medir el bienestar, si aceptamos que el bienestar de los individuos está relacionado con la facilidad con que se puede acceder a los servicios esenciales (CAF, 2017). Una accesibilidad mejorada proporciona a las personas un conjunto más amplio de oportunidades con el fin de seleccionar la que mejor se adapte a sus necesidades y preferencias. Siguiendo esta lógica, la accesibilidad puede ser considerada como un valor agregado de un lugar y un factor importante de la calidad de vida (Todd, 2014), mientras que la falta de accesibilidad es indeseable debido a que se considera en parte responsable del retraso en el desarrollo económico. 
El tipo de infraestructura de transporte determina la impedancia que es el esfuerzo (costo) necesario para cubrir la distancia entre dos puntos separados en el espacio. Esta impedancia es normalmente medida en unidades de distancia, tiempo de viaje o costo generalizado de transporte. Aspectos de seguridad, congestión o el cambio en los modos de transporte o su soporte pueden formar parte de los cambios en las medidas de impedancia (Condeço - Melhorado, 2011; Moya, 2018).

Actualmente se distinguen dos conceptualizaciones diferenciadas sobre la accesibilidad, cuya diferencia radica en el sujeto al que se aplica la cualidad de accesible (Figura 2):

El primero centra la accesibilidad individual o personal, entendida como la capacidad de movilidad individual y de acceso a servicios. Se vincula a concepciones sociales del territorio, puesto que incorpora criterios de exclusión y distancia social (Lucas, 2012; Jirón y Mansilla, 2013). Este tipo de estudios argumentan que la separación espacial tan sólo es un tipo de separación (Farrington y Farrington, 2005).

La segunda se relaciona más con el espacio concreto que con el espacio social y es la accesibilidad de los lugares. Parte de una idea más continua del espacio y distancia, aunque ésta no sea sólo distancia lineal. Se inicia con los análisis topológicos y se han complejizado incorporando otros matices. En su formulación más abstracta, combina dos elementos: la localización en una superficie en función de posibles destinos y las características de la red de transporte (Gutiérrez, 2001)

\section{Figura 2}

Diferencias entre la accesibilidad de la persona y de la ubicación o los lugares

(a)

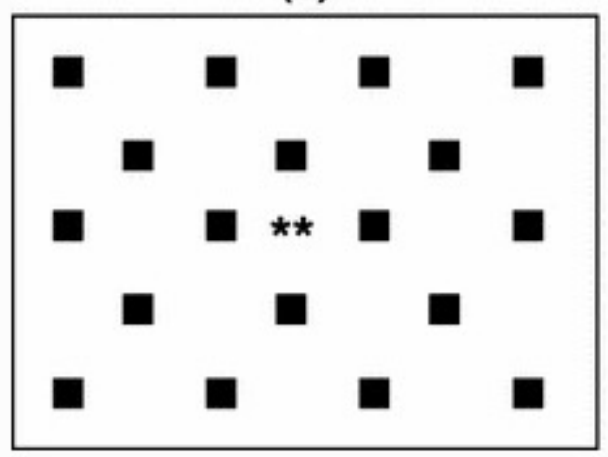

(b)

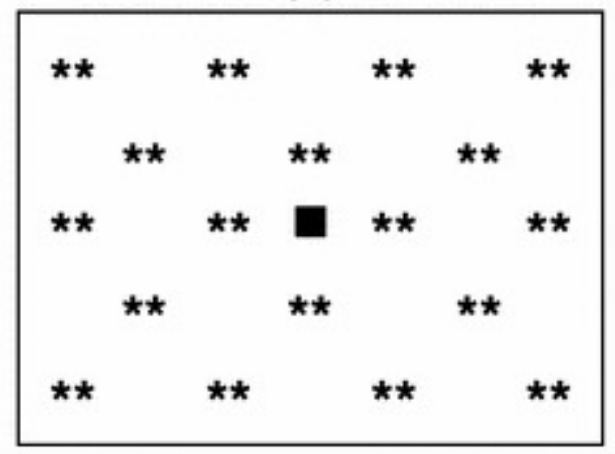

Nota: Accesibilidad de la persona (a) versus accesibilidad del lugar (b). Los bordes de cada esquema indican el área que se puede recorrer, por ejemplo, bajo un presupuesto de tiempo o costo determinado. ** = Persona, * = ubicación de la actividad. Fuente: Adaptación propia con base en Dijst, 1995, p. 28

La literatura sobre los distintos tipos de indicadores de accesibilidad y su clasificación es muy amplia y abundante (descripciones más detalladas pueden ser revisadas en Geurs y Ritsema van Eck,2001; Gutiérrez, 2004). En general, no existe un indicador único, mejor o ideal, pero se argumenta que el análisis se enriquece si un conjunto de indicadores se calcula y sus resultados se analizan de forma complementaria (Gutiérrez, 2001; Geurs y Ritsema van Eck, 2001). Así, la selección de indicadores apropiados para analizar un caso particular resulta ser una tarea compleja, ya que es frecuentemente mal entendida y definida de forma 
dispersa a través de índices excesivamente sintéticos (Geurs y Van Wee, 2004). Para poder conseguir esto, las geotecnologías resultan fundamentales, tal como se explica en adelante.

4.

\section{Las geotecnologías: una visión del es- pacio geográfico desde la informática y su aporte en los estudios de transporte, movilidad y accesibilidad}

En las últimas décadas, la aplicación de las tecnologías de la información cobra una relevancia creciente en el análisis espacial del transporte, movilidad y accesibilidad, con lo que se generan diversas plataformas basadas en sistemas de información geográfica para el transporte (GIS-T), que actualmente representan una de las aplicaciones más relevantes de los SIG y geotecnologías en el análisis espacial, tanto para resolver necesidades de investigación, como para mejorar las prácticas actuales de planificación, operación y gestión de los distintos sistemas de transporte (Shaw, 2010).

Además, los análisis geoespaciales cimentan un anhelo de la comunidad geográfica de buscar herramientas integradas de análisis espacial, que permitan vincular y complejizar los hallazgos y mediciones de modelos más estrictos de corte cualitativo o cuantitativo. Por ejemplo, con las herramientas hoy disponibles se dispone de un alto grado de flexibilidad espacial y temporal, así como la posibilidad de disponer, administrar y sistematizar grandes bases de datos, antes imposibles de poder procesar ni menos representar espacialmente.

Desde principios de la década de 2000, es posible constatar que las geotecnologías no sólo aportan en la forma en que se mueven las personas y los bienes, sino también lo que sabemos sobre estas movilidades y sus potenciales de accesibilidad. En pocos años, se pasó de la escasez de datos o la disponibilidad limitada (por los altos costos y esfuerzos en el levantamiento de información), a una avalancha de sensores y flujos de datos (Milled y Goodfchild, 2015) que proporcionan información sobre la ubicación, el momento y la frecuencia de las actividades que generan viajes (como el empleo, las compras o actividades sociales), o bien, proporcionan evidencia directa del volumen, la concentración y la dirección de los movimientos de individuos, bienes o vehículos (Milne y Watling, 2019), incluso en tiempo real.

También comienza a aportar modelos que permiten a la política pública aplicar el requerimiento teórico de cambio de paradigma del enfoque de demanda (una infraestructura en expansión) a la gestión de la demanda y oferta de infraestructuras y soportes disponibles para la movilidad (Herce, 2009), y aportar en la activación de los potenciales poco utilizados como la movilidad activa o los desplazamientos inter y multi modales. Los sistemas de apoyo al transporte multi e intermodal requieren de datos precisos y componentes de comunicación, gestión y análisis que evidentemente son espaciales (Miller y Shaw, 2015; Shaw, 2010).

También existe un cambio paradigmático donde las geotecnologías han dejado su vinculación mayoritaria con modelos estadísticos de análisis agregados, a modelos basados en actividades y microescalas, donde los comportamientos a nivel de individuos también resultan relevantes (Buliung y Kanaroglou, 2007).

Este giro en la comprensión del transporte, la movilidad y la accesibilidad basado en geotecnologías reivindica la relevancia del espacio geográfico como objeto de estudio disciplinar y campo de ocupación de la acción pública, y posibilita la integración con otras aproximaciones de la misma disciplina que buscan conciliar preocupaciones sociales a partir de enfoques epistemológicos más críticos sustentados en la producción de espacio urbano (Cidell y Prytherch, 2015).

Por último, las geotecnologías en los modelamientos de transporte y movilidad actualmente también experimentan una transición, en dondeyano se limita el uso a la presentación cartográfica de los resultados finales, sino que son herramientas poderosas que estimulan el pensamiento visual sobre patrones geoespaciales, relaciones y tendencias (Kraak, 2003). Esta potencialidad que cuenta con un vasto cuerpo de 
conceptos, metodologías y herramientas disponibles y establecidas hoy son empleados de una forma inter y multidisciplinaria, en un marco de diálogo donde la geografía como disciplina debe situarse tanto en sus procesos de formación como de aplicación.

5.

\section{Desafíos y oportunidades de la geogra- fía en su vínculo con el transporte, la movilidad y la accesibilidad}

Actualmente, nos encontramos ante un contexto que demanda entender cómo la vida social se reconfigura con nuevas tecnologías de comunicación (Sheller, 2000) y disímiles calidades en la oferta de transporte, de suelo urbano y disponibilidad de bienes y servicios urbanos que, expresan el deseo, materialización, objetividad o no, de la práctica de viajes (Gutiérrez, 2012).

Esto invita no sólo a validar, sino a potenciar el carácter inter y multidisciplinario, resultado de la naturaleza espacial del transporte y los desplazamientos, y de las estructuras y sistemas existentes que permiten o inhiben cierta movilidad.

La naturaleza aplicada de la geografía en los estudios de transporte, movilidad y accesibilidad generan una incidencia creciente en la acción pública. Hoy, la búsqueda de evidencia cuantificable, cualificable y espacializable para conducir decisiones es un desafío más vigente que nunca. Witlox y Schwanen (2019), sistematizan los principales temas que actualmente sustentan las preguntas de investigación y métodos de acción pública en estas materias.

Hoy los estudios desde la geografía dan cuenta de cómo el transporte, la movilidad y la accesibilidad son campos emergentes de la disciplina que, si bien aún no reciben un alto nivel de atención en la academia (sobre todo en términos formativos), en proporción con su relevancia económica y social (Rodrigue, 2017), hoy vislumbra avances significativos, sobre todo en la comprensión compleja de los territorios.
También es un campo que, en términos de sus metodologías, evidencia la "falsa dicotomía" entre lo cualitativo, lo cuantitativo y las geotecnologías. En los estudios de geografía del transporte, movilidad y/o accesibilidad actualmente es, cada vez, más común el desarrollo de trabajos con uso de métodos integrados, que dan cuenta de la necesidad de reconocer de mejor manera la complementariedad de enfoques y la capacidad de articular la relación entre ellos para el análisis territorial. Los actuales abordajes metodológicos dejan abierta la inquietud y necesidad de indagar en las oportunidades, amenazas e impactos de disponer de diversas fuentes de información como es hoy en Big Data, tanto para apoyar la planificación como la decisión de viajes.

De esta forma, las oportunidades para el fortalecimiento de esta subdisciplina pasan por la apertura de la geografía como disciplina y su capacidad para adaptarse y funcionar dentro de esferas multidisciplinares, espacio en que el transporte y la movilidad son focos de una gama mucho más amplia donde pueden ser incorporados otros temas como la migración, la vivienda, el medio ambiente, la salud, etc.

Por último, la geografía en este tipo de estudios hoy recupera términos que permiten plantear preguntas y respuestas relativas a los sistemas de movimiento tanto de las personas en las ciudades, como también de los bienes e información en un sistema global, pero con impactos disímiles y heterogéneos en lo local. Aspectos como la escala y la visualización del espacio, han permitido que, en la actualidad, muchos de los conceptos y métodos de la disciplina sean parte de los requerimientos mínimos del análisis espacial, que hace unas décadas era campo exclusivo de la ingeniería de transportes o del urbanismo. 


\section{Referencias}

Ascher, F. (2005). Le Mouvement dans les sociétés hypermodernes. Université de Tous Les Savois.

Bertolini, L., le Clercq, F. y Kapoen, L. (2005). Sustainable accessibility: a conceptual framework to integrate transport and land use plan-making. Two test-applications in the Netherlands and a reflection on the way forward. Transport Policy, 12, 207-220.

BID. (2014).Megaciudadese infraestructura en América Latina: lo que piensa su gente. Washington D.C: Banco Interamericano de Desarrollo.

Buliung, R., y Kanaroglou, P. (2007). Activitytravel behaviour research: Conceptual issues, state of the art, and emerging perspectives on behaviural analysis and simulation modelling. Transport Reviews, 27, 151-187.

CAF. (2017). Crecimiento urbano y acceso a oportunidades: un desafío para América Latina. Bogotá: Corporación Andina de Fomento.

Calvo-Poyo, F., Moya-Gómez, B., García Palomares, J.,y Gutiérrez, J. (2019). Efectos sobre la accesibilidad de la red de autovías planeada en el Plan de Infraestructuras para la sostenibilidad del transporte en Andalucía (España). Cuadernos geográficos, 58:1, 229-252.

Campbell, K., Rising, J., Klopp, J., y Mbilo, J. (2019). Accesibility across transport modes and residential developments in Nairobi. Journal of Transport Geography 74, 77-90.

Chen, N. y Akar, G. (2017). How do sociodemographics and built environment affect individual accessibility based on activity space? Evidence from Greater Cleveland, Ohio. Journal of Transport and Land Use, 10:1, 477-503.

Cidell, J. y Prytherch, D. (2015). Transport, Mobility and the Production of Urban Space. New York: Routledge.
Condeço- Melhorado, A., Gutiérrez, J., y GarcíaPalomares, J. (2011). Spatial impacts of road pricing: Accessibility, regional spillovers and territorial cohesion. Transportation Research Part A, 45, 185-203.

Condeço - Melhorado, A. (2011). Spatial spillovers of transport infrastructure. Alcalá de Henares: Tesis Doctoral, Departamento de Geografía, Universidad de Alcalá.

Cortés, A., Figueroa, O., y Moreno, D. (2016). Los costos del uso del automóvil y su elasticidad. El caso de Santiago de Chile. Estudios Socioterritoriales. Revista de Geografía. $N^{\circ} 20$., 2739.

Curl, A., y Davison, L. (2014). Transport Geography: perspectives upon entering an accomplished reseach sub-discipline. Journal of Transport Geography, 38., 100-105.

El-Geneidi, A., Cerdá, A., Fischler, R., y Luka, N. (2011). Evaluating the impacts of transportation plans using accessibility measures. Canadian Journal of Urban Research, Vol.20, $\mathrm{N}^{\circ} 1,81-104$.

Farrington, J., y Farrington, C. (2005). Rural accessibility, social inclusion and social justice: towards conceptualisation. Journal of Transport Geography, 13, 1-12.

Gandelman, N., Serebrisky, T., y Suárez- Alemán, A. (2019). Household spending on transport in Latin America and the Caribbean: A dimension of transport affordability in the region. Journal of Transport Geography, Vol.79, 102482.

Geurs, K., y Ritsema van Eck, J. (2001). Accessibility measures: review and applications. Evaluation of accessibility impacts of land-use transportation scenarios, and related social and economic impact. Bilthoven, Netherlands.

Geurs, K., y Van Wee, B. (2004). Accessibility evaluation of land-use and transport strategies: review and research directions. Journal of Transport Geography, 12, 127-140. 
Giménez i capdevila, R. (1986). La geografía de los transportes, en busca de su identidad. Geocrítica, $N^{\circ} 62$.

Graham, D. (2014). Causal Influence for ExPost Evaluation of Transport Interventions. International Transport Forum. Paris.

Graizbord, B. (2008). Geografía del transporte en el área Metropolitana de la Ciudad de México. Ciudad de México: Colegio de México A.C.

Gutiérrez, A. (2008). Geografía, transporte y movilidad. Espacios de Crítica y Producción, $N^{3} 37$, FFyL-UBA.

Gutiérrez, A. (2010). Movilidad, transporte y acceso: una renovación aplicada al ordenamiento territorial. XI coloquio internacional de geocrítica.

Gutiérrez,A. (2012). ¿Qué es la movilidad? Elementos para (re) construir las definiciones básicas del campo del transporte. Revista Bitácora Urbano Territorial, Vol. 21, $N^{\circ} 2$, 61-74.

Gutiérrez, J. (2001a). Escalas espaciales, escalas temporales. Estudios geográficos, Vol. 62, $N^{\circ} 242$, 92-97.

Gutiérrez, J. (2001b). Location, economic potential and daily accessibility: an analysis of the accessibility impact of the high-speed line Madrid- Barcelona- French border. Journal of Transport Geography, 9, 229-242.

Herce, M. (2009). Sobre la movilidad en la ciudad: propuestas para recuperar un derecho ciudadano. Barcelona: Reverté.

Hernández, D. (2012). Activos y estructuras de oportunidades de movilidad. Una propuesta analítica para el estudio de la accesibilidad por transporte público, el bienestar y la equidad. EURE, Vol. 38, N 115, 117-135.

Hodge, D. (1997). Accessibility-related issues. Journal of Transport Geography, 5, 33-.

Hoyle, B., y Knowles, R. (2000). Modern Transport Geography (Edition 2). New York: Wiley.
Jirón, P., y Mansilla, P. (2013). Atravesando la espesura de la ciudad: vida cotidiana y barreras de accesibilidad de los habitantes de la periferia urbana de Santiago de Chile. Revista de Geografía Norte Grande, 56, 53-74.

Kaufmann, V. (2002). Temps et pratiques modales. Le plus court est-il le mieux? The rationality of perception and modal choice. Is quickest best? Recherche Transports Sécurité, 75., 131-143.

Kaufmann, V. (2008). Les paradoxes de la mobilité. Laussane: Le Savoir Suisse.

Knowles, R., Shaw, J., y Docherty, I. (. (2008). Transport Geographies: Mobilities, Flows and Spaces. Oxford: Blackwell Publishing.

Kraak, M. J. (2003). The space-time cube revisited from a geovisualization perspective. Proceedings of a the 21st International Cartographic Conference (ICC), (pp. 1988-1995). Durban, South Africa.

Le Breton, E. (2005). Bouger pour s'en sortir. Mobilité quotidienne et intégration sociale. Paris: Armand Colin.

López, E., Ortega, E., y Condeço- Melhorado, A. (2009). Análisis de impactos territoriales del plan estratégico de infraestructuras y transporte 2005-2020: cohesión regional y efectos de desbordamiento. ICE, Aspectos territoriales del desarrollo: presente y futuro, 159-172.

López, M. J., y Ravella, O. (2019). Movilidad, espacio y diseño: metodología de análisis integrada del sistema viario en relación a sus contextos y a parámetros de diseño. El caso de la ciudad de la Plata. Revista de Urbanismo $N^{\circ}$ 40, 1-25.

Lucas, K. (2012). Transport and social exclusion: Where are we now? Transport Policy, 20, 105-113.

Martínez, H. (2012). La accesibilidad regional y el efecto territorial de las infraestructuras de transporte. Aplicación en Castilla- La Mancha. Boletín de la Asociación de Geógrafos Españoles, 59, 79-103. 
Mayhew, S. (2009). Transport Geography. In a Dictionary of Geography. Oxford: University Press.

Miralles, C. (2002). Ciudad y Transporte: el Binomio imperfecto. Barcelona: Ariel.

Módenes, J. (2007). Movilidad espacial: uso temportal de territorio y poblaciones vinculadas. $X$ Congreso de la Población Española.

Monzón, A., Ortega, E., y López, E. (2010). Social impacts of high speed rail projects: addressing spatial equity effects. 12th WCTR. Lisbon, Portugal.

Moya, B. (2018). Impacto y consecuencias de la congestión de las infraestructuras del transporte en el sistema usos del suelo- transporte- sociedad. Madrid: Tesis doctoral, Departamento de Geografía, Universidad Complutense de Madrid.

Niedzielski, M., y Boschmann, E. (2014). Travel time and distance as relative accessibility in the journey to work. Annals of the association of American Geographers, 104:6, 1156-1182.

Ortega, J. (2000). Los horizontes de la geografía. Teoría de la geografía. Barcelona: Ariel.

Páez, A., Scott, D., y Morency, C. (2012). Measuring accessibility: positive and normative implementattions of various accessibility indicators. Journal of Transport Geography, 141153.

Potrykowski, M., y Taylor, Z. (1984). Geografía del transporte. Barcelona: Ariel.

Rodrigue, J.-P. (2017). The geography of transport systems. New York: Routledge.

Seguí, J., y Petrus, J. (1991). Geografía de redes y sistema de transporte. Madrid: Síntesis S.A.

Shaw, S. (2010). Geographic information systems for transportation: from a static past to a dynamic future. Annals of GIS, 16:3, 129-140.
Silva, C., Bertolini, L., Brömmelstroet, M., Milakis, D., y Papa, E. (2017). Accesibility instruments in planning practice: bridging the implementation gap. Transport Policy, 135-145.

Todd, L. (2014). Transportation and the Quality of Life. En M. A. (Eds), Encyclopedia of quality of life and well-being research. Dordrecht: Springer.

UN Habitat. (2016). World Cities Report: Urbanization and Development: Emerging Futures.

Urry, J. (2002). Mobility and proximity. Sociology, $\mathrm{N}^{\circ} 36,255-274$.

Widmer, E., y Schneider, N. (. (2006). State of the art of Mobility Research. A literature analysis for eight countries. EU: Job Mobilities Working Paper, 2006-1. EU: Job Mob and Fam Lives.

Witlox, F., y Schwanen, T. (2019). Looking back, to move forward: celebrating 25 years Journal of Transport Geography. Journal of Transport Geography, Vol. 81, 102591.

Zondag, B., de Bok, M., Geurs, K., y Molenwijk, E. (2015). Accessibility modeling and evaluation: the TIGRIS XL land-use and transport interaction model for the Netherlands. Computers, Environment and Urban Systems, 49, 115-125. 
